\title{
Erratum to: First molecular detection of porcine circovirus type 2 in bovids in China
}

\author{
Shao-Lun Zhai ${ }^{1,2}$ - Rui-Ai Chen ${ }^{1,3}$ - Sheng-Nan Chen ${ }^{3}$ Xiao-Hui Wen ${ }^{2}$. \\ Dian-Hong $\mathrm{Lv}^{2} \cdot$ Da-Cheng $\mathrm{Wu}^{2} \cdot$ Jie Yuan ${ }^{2} \cdot$ Zhong Huang ${ }^{2} \cdot$ Xiu-Rong $\mathrm{Zhou}^{2}$ • \\ Man-Lin Luo ${ }^{1,3} \cdot$ Dong-Sheng $\mathrm{He}^{1,3} \cdot$ Wen-Kang Wei ${ }^{2}$
}

Published online: 17 November 2015

(C) Springer Science+Business Media New York 2015

\section{Erratum to: Virus Genes (2014) 49:507-511 \\ DOI 10.1007/s11262-014-1117-1}

Unfortunately, we have noticed an error in Materials and methods section of the published article. Instead of "The expected product size was 496 bp (targeted ORF2 genes: position 1096 to 1587 or 1588)", it should be "The expected product size was 493 bp (targeted ORF2 genes: position 1095 to 1587 or 1096 to 1588$) "$.

The online version of the original article can be found under doi:10.1007/s11262-014-1117-1.

Man-Lin Luo

luoml@scau.edu.cn

$\triangle$ Dong-Sheng He

dhe@scau.edu.cn

$\triangle$ Wen-Kang Wei

wwk188@tom.com

1 College of Veterinary Medicine, South China Agricultural University, Guangzhou 510642, China

2 Institute of Animal Health, Guangdong Academy of Agricultural Sciences, Guangzhou 510640, China

3 Guangdong Dahuanong Animal Health Products Co., Ltd., Xinxing 527400, China 